\title{
Optimal Locations and the Mass Transport Problem
}

\author{
Michael McAsey and Libin Mou \\ Department of Mathematics \\ Bradley University \\ Peoria, IL 61625 \\ mcasey@bradley.edu,mou@bradley.edu
}

\section{Introduction}

Suppose the measures $\mu$ and $\nu$ represent two mass distributions on $R^{m}$ of equal total measure. Let $c(x, y)$ be the cost (per unit mass) for transporting the mass from $x$ to $y$. A transport plan is a measure $\gamma$ on the product $R^{m} \times R^{m}$ with marginals $\mu$ and $\nu$, with total cost

$$
\int_{R^{m} \times R^{m}} c(x, y) d \gamma
$$

The Monge-Kantorovich mass transport problem is to find the transport plan that minimizes the cost (1). This was formulated by Kantorovich in the 1940's, generalizing Monge's format in 1781. Monge's original formulation of the problem assumes that $c(x, y)=|x-y|$ and asks for the existence of a mass-preserving map $f$ from the support, $\operatorname{Spt}(\mu)$, of $\mu$ to $\operatorname{Spt}(\nu)$ that minimizes the cost

$$
\int_{R^{m}} c(x, f(x)) d \mu
$$

This is a special case of (1) when $\gamma$ is the measure supported on the graph of $f$ with marginals $\mu$ and $\nu$. Since the functional (1) is linear in $\gamma$ and the set of $\gamma$ 's whose marginals are $\mu$ and $\nu$ is a convex subset, a minimum of (1) exists under general conditions on $\mu$ and $c$; see [8], [13] for examples. While Monge's definition of a transport plan appears to be more natural, the existence of a minimum for (2) requires several conditions. First, to ensure the existence of a mass-preserving map from $\operatorname{Spt}(\mu)$ to $\operatorname{Spt}(\nu)$, the measure $\mu$ should not be concentrated on small sets. Second, some sort of concavity of the cost function $c$ is needed to assert the uniqueness of the minimum. See [8] and the references therein.

The problem of mass transport can also be phrased in the language of probability. In this connection, the optimal mass transport problem corresponds to optimal coupling of random variables. Results in one context can be interpreted under the other. See [10], [11], [13]for examples. The authors wish to thank the referee for pointing out several significant references. 
Many real-life problems can be modeled by mass transport problems. Consider, for example, the problem of finding locations of waste management facilities in a city. Other examples can be found in [12], [13], [14].

In many cases, however, the source measure $\mu$ is known but the target measure $\nu$ is only partially known, and the problem is to find the location of $\operatorname{Spt}(\nu)$ and the transport plan so that the cost is minimum. This is especially true in location problems, see [2], [3], [9], [14], [16], [18] for examples. In this paper we show the existence of $\operatorname{Spt}(\nu)$ such that the total cost of transporting a source mass $\mu$ to $\operatorname{Spt}(\nu)$ is minimal among a class of admissible target measures.

We first consider the case that the target measure $\nu$ is supported on a finite number points $z_{1}, \ldots, z_{n}$ (to be determined) with prescribed positive masses $\alpha_{1}, \ldots, \alpha_{n}$ with $\sum_{i=1}^{n} \alpha_{i}=1$. That is, we assume

$$
\nu=\sum_{i=1}^{n} \alpha_{i} \delta_{z_{i}}
$$

where $\delta_{z_{i}}$ is the point mass concentrated at $z_{i}$. (As applied to mass transport problems, the $\alpha_{i}$ can be thought of as the capacity constraint for a facility located at $z_{i}$.)

Assuming that $z_{1}, \ldots, z_{n}$ have been found and that $\mu$ does not concentrate on certain sets of lower "dimensions", then an optimal transport plan exists, which can berepresented by a map $P$ from $\operatorname{Spt}(\mu)$ to $\operatorname{Spt}(\nu)=\left\{z_{1}, \ldots, z_{n}\right\}$. The map $P$ generates a partition $D_{1}, \ldots, D_{n}$ of $\operatorname{Spt}(\mu)$, given by $D_{i}=P^{-1}\left(z_{i}\right), i=1, \ldots, n$. (The transport plan is given by "transport the mass in $D_{i}$ to the location $z_{i}$.") The total cost (2) can be written as

$$
F\left(z_{1}, \ldots, z_{n}, D_{1}, \ldots, D_{n}\right)=\sum_{i=1}^{n} \int_{D_{i}} c\left(x, z_{i}\right) d \mu
$$

This result is proved by Abdellaoui [1], Cuesta-Albertos and Tuero-Diaz [6], Gangbo and McCann [8], and Ruschendorf [10], [11].

Our first problem can now be stated as follows. Given

$$
\alpha_{1}, \ldots, \alpha_{n}>0 \text {, with } \alpha_{1}+\cdots+\alpha_{n}=1,
$$

find locations $z_{1}, \ldots, z_{n}$ in $R^{m}$ and a partition $D_{1}, \ldots, D_{n}$ of the support of $\mu$ such that

$$
\mu\left[D_{i}\right]=\int_{D_{i}} d \mu=\alpha_{i}, i=1, \ldots, n
$$

and the functional (4) is minimal among all locations and partitions satisfying (6). 
In Theorem 2 below, we prove the existence of optimal $z_{1}, \ldots, z_{n}$ and $D_{1}, \ldots, D_{n}$ under fairly general conditions on $\mu$ and $c$. As an important part of the proof of Theorem 2 , we need Theorem 1, which is a characterization of the optimal partitions $D_{1}, \ldots, D_{n}$ of (4) for given $z_{1}$, $\ldots, z_{n}$. In addition, we need to analyze the limit behaviors of a minimizing sequence $z_{1}, \ldots, z_{n}$ and the corresponding sequence of optimal partitions $D_{1}, \ldots, D_{n}$; some techniques of this part are similar to those in the paper of Cuesta-Albertos [4].

There are conditions on $\mu$ and $c(x, y)$ for which Theorem 1 is well-known (cf. [1], [8], [11]. Our proof of Theorem 1 is somewhat long but is straightforward and might be of interest in its own right.

In Section 3, we consider the problem for which the target measure $\nu$ is supported on a subset $M$ of $R^{m}$. Here we do not make any assumptions on how the resulting mass is distributed on $M$. Therefore, the optimal transport plan is simple: the mass at the $x \in \operatorname{Spt}(\mu)$ is transported to a point in $M$ such that $c(x, y) \leq c(x, z)$ for all $z \in M$. The total cost of transferring the mass $\mu$ to $M$ is

$$
F(M)=\int_{R^{n}} c(x, M) d \mu(x),
$$

where $c(x, M)$ is the cost of transferring a unit mass from $x$ to $M$. Note that $c(x, M)=\min _{y \in M}$ $c(x, y)$.

When the cost function $c(x, y)=|x-y|^{2}$, then the optimal $M$ is equivalent to the selfconsistent set (points, curve, or surface) of a distribution, see [15, Definition 6.1] and the references therein. When the cost function is a more general function of the norm $|x-y|$, the optimal set $M$ of $k$ points is called the $k$-means as in [4], [5]. The concept of self-consistency is fundamental in statistics and it has applications in various fields including signal transmissions; see [15].

The existence of an optimal set $M$ of a finite number of points has been proved in [4], [5] and [12]. In Section 3, we prove the existence of $M$ that gives the minimal cost $F$ in classes of compact sets of arbitrary dimensions.

\section{Existence of Optimal Locations - the finite point case.}

In this section, we will give a direct proof (without using convex analysis) of the existence of an optimal plan for measures $\nu$ of the form (3) under mild conditions on $\mu$ and $c(x, z)$. 
Theorem 1 shows how, given $n$ points $z_{1}, \ldots, z_{n}$, we can construct an optimal partition $D_{1}, \ldots, D_{n}$. This is a technical result but gives an explicit description of the sets $D_{i}$. The form of these sets is suggested in Example 1.6 of [8]. For the cost $c(x, y)=|x-y|^{p}$, Theorem 1 is also proved in [1]. Then in Theorem 2 we show that the support of $\nu, \operatorname{Spt}(\nu)=\left\{z_{1}, \ldots, z_{n}\right\}$, can be chosen such that total transportation cost (4) is minimal among all possible points $z_{1}, \ldots, z_{n}$ in $R^{m}$ and partitions $D_{1}, \ldots, D_{n}$ satisfying (6).

Theorem 1. Suppose $c(x, z): R^{m} \times R^{m} \rightarrow[0, \infty)$ is a continuous function, $z_{1}, \ldots, z_{n}$ in $R^{m}$ are given points and $\alpha_{1}, \ldots, \alpha_{n}$ are positive numbers so that $\alpha_{1}+\cdots+\alpha_{n}=1$. Suppose $\mu$ is a regular Borel probability measure such that for any $z_{i}, z_{j}$ and constant $\lambda$,

$$
\mu\left\{x \in \operatorname{Spt}(\mu): c\left(x, z_{i}\right)-c\left(x, z_{j}\right)=\lambda\right\}=0 .
$$

Then

(a) there exist $\lambda_{1}, \ldots, \lambda_{n}$ such that the partition

$$
D_{i}=\left\{x: c\left(x, z_{i}\right)+\lambda_{i} \leq c\left(x, z_{j}\right)+\lambda_{j}, j \neq i\right\}
$$

satisfies $\mu\left[D_{i}\right]=\alpha_{i}$.

(b) Among all partitions $E_{1}, \ldots, E_{n}$ satisfying (6), the partition $D_{1}, \ldots, D_{n}$ defined in (8) is optimal, i.e., it minimizes the total cost (4) for the given $z_{1}, \ldots, z_{n}$.

Note that (7) says that any boundary between two regions $D_{i}$ and $D_{j}$ has zero measure. This condition was also used in [6]. Also note that the sets $D_{i}$ defined in (8) depend on the numbers $\lambda_{i}$. Theorem 1(a) says that it is possible to choose $\lambda=\left(\lambda_{1}, \ldots, \lambda_{n}\right)$ so that $\mu\left[D_{i}\right]=\alpha_{i}$ for all $i=1, \ldots, n$. Clearly it is easy to find $\lambda$ and associated partitions of $R^{m}$ that satisfy this equation for some $i$ but not necessarily for all $i$. In particular one of these $\lambda$ 's can be specified arbitrarily and then the others need to be chosen carefully. Showing that all the required $\lambda$ 's can be chosen simultaneously occupies most of the proof.

To prove Theorem 1(a) we will use induction on the number of those $i$ 's for which $\mu\left[D_{i}\right]=\alpha_{i}$. We will let $\lambda=\left(\lambda_{1}, \ldots, \lambda_{n}\right)$ and $f_{i}(\lambda)=\mu\left[D_{i}\right]$. For $k=1, \ldots, n$, we let

$$
S_{\left\{\alpha_{1}, \ldots, \alpha_{k}\right\}}=\left\{\lambda: f_{i}(\lambda)=\alpha_{i}, i=1, \ldots, k\right\} .
$$

The sets $S_{\left\{\alpha_{1}, \ldots \alpha_{k}\right\}}$ measure how close we are to finding $\lambda$ 's that describe a partition satisfying the required condition (6). So for Theorem 1(a) we need to show that $S_{\left\{\alpha_{1}, \ldots, \alpha_{n}\right\}} \neq \emptyset$. Because $\alpha_{1}+\cdots+\alpha_{n}=1$, we need only show that $S_{\left\{\alpha_{1}, \ldots, \alpha_{n-1}\right\}} \neq \emptyset$. This will be a special case of the following Main Lemma, which says that $S_{\left\{\alpha_{1}, \ldots, \alpha_{k}\right\}} \neq \emptyset$ for any $k \leq n-1$. We say that a 
measure is strongly positive if the measure of any measurable set with non-empty interior is positive.

\section{Main Lemma}

(i) For $i=1, \ldots, n, f_{i}\left(\lambda_{1}, \ldots, \lambda_{n}\right)$ is continuous in $\lambda$.

(ii) Suppose $\mu$ is strongly positive. For any $k \in\{0,1, \ldots, n-1\}$, if $\left(\lambda_{k+1}, \ldots, \lambda_{n}\right) \in R^{n-k}$, then there exists unique $\left(\lambda_{1}^{*}, \ldots, \lambda_{k}^{*}\right)$ determined by $\left(\lambda_{k+1}, \ldots, \lambda_{n}\right)$ such that $f_{i}\left(\lambda_{1}^{*}, \ldots, \lambda_{k}^{*}\right.$, $\left.\lambda_{k+1}, \ldots, \lambda_{n}\right)=\alpha_{i}$ for each $i=1, \ldots, k$.

The proof of this lemma is rather technical and depends in turn on several other lemmas. The central job of these lemmas is to explore the functions $f_{i}$ (as functions of the $\lambda$ 's) and to show that we can solve for some of the $\lambda$ 's in terms of the others. The strong positivity assumption guarantees the uniqueness of the $\lambda^{*}$ 's. To avoid obscuring the proof of Theorem 1 , these results are stated and proved in Section 4. Finally note that the statement of Theorem 1 does not require that the measure $\mu$ satisfy the strongly positive hypothesis. To be able to apply the Main Lemma we perturb $\mu$ with a strongly positive measure.

Proof of Theorem 1. (a) Let $\mu_{0}$ be a strongly positive absolutely continuous probability measure. Consider $\mu_{\epsilon}=(1-\epsilon) \mu+\epsilon \mu_{0}$ for $\epsilon \in(0,1)$. Let $\lambda_{n}=0$. (Any fixed value for $\lambda_{n}$ would work as well.) Applying the Main Lemmato $\mu_{\epsilon}$ with $k=n-1$, there exists $\left(\lambda_{1, \epsilon}^{*}, \ldots, \lambda_{n-1, \epsilon}^{*}\right)$ such that $f_{i, \epsilon}\left(\lambda_{1, \epsilon}^{*}, \ldots, \lambda_{n-1, \epsilon}^{*}, 0\right)=\mu_{\epsilon}\left[D_{i}\right]=(1-\epsilon) \mu\left[D_{i}\right]+\epsilon \mu_{0}\left[D_{i}\right]=\alpha_{i}, i=1, \ldots, n-1$, and $D_{i}$ is defined by (8) using the parameters $\left(\lambda_{1, \epsilon}^{*}, \ldots, \lambda_{n-1, \epsilon}^{*}, 0\right)$. Since $\sum_{i=1}^{n} f_{i, \epsilon}\left(\lambda_{1, \epsilon}^{*}, \ldots, \lambda_{n-1, \epsilon}^{*}, 0\right)=\sum_{i=1}^{n} \alpha_{i}=1$, then $f_{n, \epsilon}\left(\lambda_{1, \epsilon}^{*}, \ldots, \lambda_{n-1, \epsilon}^{*}, 0\right)=\alpha_{n}$. We claim that when $\epsilon \rightarrow 0$, the net $\left\{\left(\lambda_{1, \epsilon}^{*}, \ldots, \lambda_{n-1, \epsilon}^{*}\right)\right\}_{\epsilon}$ must be bounded. For otherwise, by passing to a subnet (and rearranging components if necessary), we may assume that $\lambda_{1, \epsilon}^{*} \rightarrow+\infty$ or $-\infty$ as $\epsilon \rightarrow 0$. If $\lambda_{1, \epsilon}^{*} \rightarrow+\infty$, we would have

$$
\begin{aligned}
& \alpha_{1}=f_{1, \epsilon}\left(\lambda_{1, \epsilon}^{*}, \ldots, \lambda_{n-1, \epsilon}^{*}, 0\right) \\
& =\mu_{\epsilon}\left[\left\{x: c\left(x, z_{1}\right)+\lambda_{1, \epsilon}^{*} \leq c\left(x, z_{j}\right)+\lambda_{j, \epsilon}^{*}, j=2, \ldots, n\right\}\right] \\
& \leq \mu_{\epsilon}\left[\left\{x: c\left(x, z_{1}\right)+\lambda_{1, \epsilon}^{*} \leq c\left(x, z_{n}\right)\right\}\right] \\
& \leq \mu\left[\left\{x: c\left(x, z_{1}\right)+\lambda_{1, \epsilon}^{*} \leq c\left(x, z_{n}\right)\right\}\right]+\epsilon \rightarrow 0
\end{aligned}
$$

as $\epsilon \rightarrow 0$, contradicting $\alpha_{1}>0$. Similarly, if $\lambda_{1, \epsilon}^{*} \rightarrow-\infty$, then 


$$
\begin{aligned}
& \alpha_{n}=f_{n, \epsilon}\left(\lambda_{1, \epsilon}^{*}, \ldots, \lambda_{n-1, \epsilon}^{*}, 0\right) \\
& \leq \mu_{\epsilon}\left[\left\{x: c\left(x, z_{n}\right) \leq c\left(x, z_{1}\right)+\lambda_{1, \epsilon}^{*}\right\}\right] \\
& \leq \mu\left[\left\{x: c\left(x, z_{n}\right) \leq c\left(x, z_{1}\right)+\lambda_{1, \epsilon}^{*}\right\}\right]+\epsilon \rightarrow 0
\end{aligned}
$$

as $\epsilon \rightarrow 0$. This contradicts $\alpha_{n}>0$.

Now knowing that the net $\left\{\left(\lambda_{1, \epsilon}^{*}, \ldots, \lambda_{n-1, \epsilon}^{*}\right)\right\}_{\epsilon}$ is bounded, we may assume that $\left(\lambda_{1, \epsilon}^{*}, \ldots, \lambda_{n-1, \epsilon}^{*}\right) \rightarrow\left(\lambda_{1}^{*}, \ldots, \lambda_{n-1}^{*}\right)$ as $\epsilon \rightarrow 0$. Part (i) of the Main Lemma implies $f_{i, \epsilon}$ is continuous with respect to $\lambda$ and $\epsilon$ and so

$$
f_{i}\left(\lambda_{1}^{*}, \ldots, \lambda_{n-1}^{*}, 0\right)=\lim _{\epsilon \rightarrow 0} f_{i, \epsilon}\left(\lambda_{1, \epsilon}^{*}, \ldots, \lambda_{n-1, \epsilon}^{*}, 0\right)=\alpha_{i} .
$$

This shows part (a).

(b) Suppose $E_{1}, \ldots, E_{n}$ is any partition of $\operatorname{Spt}(\mu)$ satisfying (6). Then by the definition (8) of $D_{1}, \ldots, D_{n}$

$$
\begin{aligned}
F\left(z_{1}, \ldots, z_{n}, E_{1}, \ldots, E_{n}\right) & =\sum_{j=1}^{n} \int_{E_{j}} c\left(x, z_{j}\right) d \mu \\
& =\sum_{j=1}^{n} \sum_{i=1}^{n} \int_{E_{j} \cap D_{i}} c\left(x, z_{j}\right) d \mu \\
& \geq \sum_{i=1}^{n} \sum_{j=1}^{n} \int_{E_{j} \cap D_{i}}\left(c\left(x, z_{i}\right)+\lambda_{i}-\lambda_{j}\right) d \mu \\
& =\sum_{i=1}^{n} \int_{D_{i}} c\left(x, z_{i}\right) d \mu+\sum_{i=1}^{n} \sum_{j=1}^{n}\left(\lambda_{i}-\lambda_{j}\right) \mu\left(E_{j} \cap D_{i}\right) \\
& =\sum_{i=1}^{n} \int_{D_{i}} c\left(x, z_{i}\right) d \mu+\sum_{i=1}^{n} \lambda_{i} \mu\left(D_{i}\right)-\sum_{j=1}^{n} \lambda_{j} \mu\left(E_{j}\right) \\
& =\sum_{i=1}^{n} \int_{D_{i}} c\left(x, z_{i}\right) d \mu+0=F\left(z_{1}, \ldots, z_{n}, D_{1}, \ldots, D_{n}\right) .
\end{aligned}
$$

Thus the total cost function is minimized with the partition $D_{1}, \ldots, D_{n}$ defined in (8).

Now that we know a suitable partition exists for arbitrary choices of points $z_{1}, \ldots, z_{n}$, we want to show that it is possible to choose these points to minimize the total cost (4). This is the first of the existence theorems of the paper. The hypotheses on the cost function $c(x, z)$ in 
Theorem 2 ( and Theorem 3) are supposed to suggest properties of a per unit transportation cost between points $x$ and $z$ in applications.

\section{Theorem 2. Suppose}

(i) $c(x, z): R^{m} \times R^{m} \rightarrow[0, \infty)$ is continuous in both $x$ and $z$ with

$$
c_{0}=\sup \left\{c(x, z):(x, z) \in R^{m} \times R^{m}\right\} \in(0, \infty]
$$

such that for any compact set $E \subset R^{m}$,

$$
\begin{gathered}
\max \{c(x, z):(x, z) \in E \times E\}<c_{0} \\
\min \{c(x, z): x \in E\} \rightarrow c_{0} \text { as }\|z\| \rightarrow \infty ;
\end{gathered}
$$

(ii) $\mu$ is a regular Borel probability measure such that for all points $z_{1}, z_{2}$ and constant $\lambda$,

$$
\mu\left\{x \in \operatorname{Spt}(\mu): c\left(x, z_{1}\right)-c\left(x, z_{2}\right)=\lambda\right\}=0 .
$$

Then there exist points $z_{1}, \ldots, z_{n}$ and a partition $D_{1}, \ldots, D_{n}$ of $\operatorname{Spt}(\mu)$ such that the total cost is minimal among all locations and partitions satisfying (8).

Proof. For any collection of points $z_{1}, \ldots, z_{n}$, Theorem 1 guarantees the existence of a partition $D_{1}, \ldots, D_{n}$ defined using these points as in (8) and satisfying the requirements of (6).

Furthermore, this partition gives a minimum value for the total cost $F$ for all partitions satisfying (6). Thus we can regard $F$ as a function of the points $z_{1}, \ldots, z_{n}$ and write

$$
F\left(z_{1}, \ldots, z_{n}\right)=\sum_{i=1}^{n} \int_{D_{i}} c\left(x, z_{i}\right) d \mu .
$$

We now show that there is a choice of $z_{1}, \ldots, z_{n}$ that minimizes $F\left(z_{1}, \ldots, z_{n}\right)$. First note that assumption (i) implies that for any $z_{1}, \ldots, z_{n}$,

$$
F\left(z_{1}, \ldots, z_{n}\right)<c_{0} .
$$

This shows that the greatest lower bound $F_{\min }$ of $F$ among all possible $z_{1}, \ldots, z_{n}$ exists and satisfies

$$
F_{\min }<c_{0}
$$

The rest of the proof consists of showing that $F_{\min }$ is achieved at some $z_{1}^{0}, \ldots, z_{n}^{0}$.

Take a minimizing sequence $\left\{z_{1}^{k}, \ldots, z_{n}^{k}\right\}_{k=1}^{\infty}$ such that $F\left(z_{1}^{k}, \ldots, z_{n}^{k}\right) \rightarrow F_{\min }$ as $k \rightarrow \infty$. Let $D_{1}^{k}, \ldots, D_{n}^{k}$ be the partition and $\lambda_{1}^{k}, \ldots, \lambda_{n}^{k}$ be the parameters as in (8), that are associated 
with $z_{1}^{k}, \ldots, z_{n}^{k}$; that is,

$$
D_{i}^{k}=\left\{x: c\left(x, z_{i}^{k}\right)+\lambda_{i}^{k} \leq c\left(x, z_{j}^{k}\right)+\lambda_{j}^{k} \text { for all } j \neq i\right\}
$$

The following assertion will allow extraction of convergent subsequences of $\left\{\lambda_{i}^{k}\right\}_{k}$ and of $\left\{z_{i}^{k}\right\}_{k}$.

Assertion. For all $i \in\{1, \ldots, n\}$,

(i) $\left\{\lambda_{i}^{k}\right\}_{k}$ can be chosen to be bounded.

(ii) $\left\{z_{i}^{k}\right\}_{k}$ has a bounded subsequence.

The proof of this assertion can be found in the Section 5. Assuming the assertion, we continue the proof.

By passing to a subsequence, we may assume that for all $i \in\{1, \ldots, n\}$, we have $\lambda_{i}^{k} \rightarrow \lambda_{i}^{0}$ and also $z_{i}^{k} \rightarrow z_{i}^{0}$, as $k \rightarrow \infty$.

Let $D_{1}^{0}, \ldots, D_{n}^{0}$ be defined as in (8) with $\lambda_{1}^{0}, \ldots, \lambda_{n}^{0}$ and $z_{1}^{0}, \ldots, z_{n}^{0}$. Note that the interior $\operatorname{Int}\left(D_{i}^{0}\right)$ of $D_{i}^{0}$ can be expressed as

$$
\operatorname{Int}\left(D_{i}^{0}\right)=\left\{x: c\left(x, z_{i}^{0}\right)+\lambda_{i}^{0}<c\left(x, z_{j}^{0}\right)+\lambda_{j}^{0} \text { for all } j \neq i\right\} .
$$

This is a strengthened version of (8). We show next that in fact $D_{1}^{0}, \ldots, D_{n}^{0}$ satisfy the requirements of the target measure (8). Define intersections and unions of $D_{i}^{k}$ by

$$
E_{i m}=\cap_{k=m}^{\infty} D_{i}^{k} \text { and } E_{i 0}=\cup_{m=1}^{\infty} E_{i m}
$$

Then by (12) and (13), it is easy to verify that for each $i=1, \ldots, n$

$$
\operatorname{Int}\left(D_{i}^{0}\right) \subset E_{i 0} \subset D_{i}^{0}
$$

(If $x \in \operatorname{Int}\left(D_{i}^{0}\right)$, then by (13), $x \in E_{i m}$ for all large $m$, which implies that $x \in E_{i 0}$. If $x \in E_{i 0}$, then $x \in E_{i m}$ for some $m$, which implies that $x \in D_{i}^{k}$ for all $k \geq m$. Taking the limit in (12), we see that $x \in D_{i}^{0}$.) Assumption (ii) implies that $\mu\left[\operatorname{Int}\left(D_{i}^{0}\right)\right]=\mu\left[D_{i}^{0}\right]=\alpha_{i}$. So $D_{1}^{0}, \ldots, D_{n}^{0}$ 
satisfy (6). Letting $\chi_{E}$ be the characteristic function of the set $E$, we have

$$
\begin{aligned}
\sum_{i=1}^{n} \int_{D_{i}^{0}} c\left(x, z_{i}^{0}\right) d \mu & =\sum_{i=1}^{n} \int_{\operatorname{Int}\left(D_{i}^{0}\right)} c\left(x, z_{i}^{0}\right) d \mu \\
& \leq \sum_{i=1}^{n} \int_{R^{m}} c\left(x, z_{i}^{0}\right) \chi_{E_{i 0}}(x) d \mu
\end{aligned}
$$

By (14), we see that $\chi_{E_{i k}}(x) \rightarrow \chi_{E_{i 0}}(x)$ as $k \rightarrow \infty$. It follows that $c\left(x, z_{i}^{k}\right) \chi_{E_{i k}}(x) \rightarrow c\left(x, z_{i}^{0}\right) \chi_{E_{i 0}}(x)$. By (15) and Fatou's lemma,

$$
\begin{aligned}
F\left(z_{1}^{0}, \ldots, z_{n}^{0}\right) & =\sum_{i=1}^{n} \int_{D_{i}^{0}} c\left(x, z_{i}^{0}\right) d \mu \\
& \leq \sum_{i=1}^{n} \int_{R^{m}} c\left(x, z_{i}^{0}\right) \chi_{E_{i 0}}(x) d \mu \\
& \leq \liminf _{k \rightarrow \infty} \sum_{i=1}^{n} \int_{R^{m}} c\left(x, z_{i}^{k}\right) \chi_{E_{i k}} d \mu \\
& \leq \liminf _{k \rightarrow \infty} \sum_{i=1}^{n} \int_{D_{i}^{k}} c\left(x, z_{i}^{k}\right) d \mu \\
& =F_{\min }
\end{aligned}
$$

This shows that $F$ has a minimum at $z_{1}^{0}, \ldots, z_{n}^{0}$. 


\section{Existence of Optimal Locations of Higher Dimensions}

In this section, we consider target measures $\nu$ that are supported on sets $M$ in $R^{m}$ of higher dimensions. The mass of $\mu$ at $x \in \operatorname{Spt}(\mu)$ is transported to a point in $M$ so as to minimize the unit cost:

$$
c(x, M)=\min _{y \in M} c(x, y) .
$$

The total cost of transferring the mass $\mu$ to $M$ is:

$$
F(M)=\int_{R^{m}} c(x, M) d \mu(x) .
$$

The goal is to find $M$ (in a specified class of sets $\mathcal{M}$ ) to minimize $F(M)$. Note that the distribution of $\nu$ is not prescribed. It is determined by the transport plan, which may not be unique. So the distribution of $\nu$ is not necessarily unique.

To state Theorem 3, we need assumptions on the class $\mathcal{M}$ of allowable sets and the cost function $c$. These assumptions are broken into two groups. Recall that the diameter of a set $D \subset R^{m}$ is the number $\operatorname{diam}(D)=\max \{\|x-y\|, x, y \in D\}$.

Assumption 1. Suppose $\mathcal{M}$ is a collection of bounded and closed subsets of $R^{m}$ such that

(i) If $M \in \mathcal{M}$, then $\mathcal{M}$ contains the union of any of the connected components of $M$.

(ii) If $\left(M_{k}\right)$ is a sequence in $\mathcal{M}$ and $M_{k} \rightarrow M_{0}$ in Hausdorff distance, then $M_{0} \in \mathcal{M}$.

(iii) There is a fixed bound $d_{0}>0$ for the diameter of any connected component $D$ of a set $M \in \mathcal{M}$ :

$$
\operatorname{diam}(D) \leq d_{0}
$$

\section{Assumption 2.}

(i) $c(x, z): R^{m} \times R^{m} \rightarrow[0, \infty)$ is continuous in both $x$ and $z$ with

$$
c_{0}=\sup \left\{c(x, z):(x, z) \in R^{m} \times R^{m}\right\} \in(0, \infty]
$$

such that for any compact set $E \subset R^{m}$,

$$
\begin{gathered}
\max \{c(x, z):(x, z) \in E \times E\}<c_{0} \\
\min \{c(x, z): x \in E\} \rightarrow c_{0} \text { as }\|z\| \rightarrow \infty .
\end{gathered}
$$

(ii) $\mu$ is a regular Borel probability measure with compact support. 
Some examples of classes of sets $\mathcal{M}$ are given next. It is not difficult to check that these examples satisfy Assumption 1. Suppose $N$ is a positive integer and $L$ is a positive number.

(1) $\mathcal{M}=\left\{M: M \subset R^{m}\right.$ with $\left.|M| \leq N\right\}$, where $|M|$ is the cardinality of $M$. This was considered previously in [4], [5], [12].

(2) $\mathcal{M}=\left\{M: M\right.$ is a line segment in $R^{m}$ of length $\left.\leq L\right\}$ or $\mathcal{M}=\{M: M$ is a union of at most $N$ segments of total length $\leq L\}$.

(3) $\mathcal{M}=\left\{M: M\right.$ is the image of a map from $[0,1]$ to $R^{m}$ with $\left.\left\|f^{\prime}\right\|_{\infty} \leq L\right\}$, where $\left\|f^{\prime}\right\|_{\infty}$ is the maximum norm of $\left|f^{\prime}\right|$.

(4) $\mathcal{M}=\left\{M: M\right.$ is a continuumsuch that $\left.H^{1}(M) \leq L\right\}$, where $H^{1}$ is the Hausdorff measure of dimension one. Or we can consider $\mathcal{M}=\{M: M$ is the union of at most $N$ continua with $\left.H^{1}(M) \leq L\right\}$. By definition, a continuum is a connected compact set. The property (ii) of Assumption 1 is due to the lower semicontinuity of $H^{1}$; see [7, Theorem 3.18].

(5) For any integer $s \in[1, n]$, let

$$
\mathcal{M}=\left\{M: M \text { is a continuum such that } V^{i}(M) \leq L, i=1, \ldots, s\right\},
$$

where $V^{i}$ is the $i$-variation, which is the $i$-dimensional volume for smooth surfaces generalized to compact sets [17]. Vitushkin's semicontinuity theorem [17] implies that $\mathcal{M}$ is closed with respect to Hausdorff distance. More generally, the set

$\mathcal{M}=\left\{M: M\right.$ is a union of at most $N$ continua with $\left.V^{i}(M) \leq L, i=1, \ldots, s\right\}$ satisfies the Assumption 1.

Theorem 3. Assumption 1 on $\mathcal{M}$ and Assumption 2 on $\mu$ imply that $F$ has a minimum among all $M$ 's in $\mathcal{M}$.

The proof follows along the same lines as the proof of Theorem 2. We need the lower semi-continuity of $F$ which is proven next. Let $H(A, B)$ denote the Hausdorff distance between the sets $A$ and $B$; see [7].

Lemma 6. The cost function $F(M)$ is lower semi-continuous with respect to Hausdorff distance, that is, if $M_{k}, M_{0} \in \mathcal{M}$ and $H\left(M_{k}, M_{0}\right) \rightarrow 0$ as $k \rightarrow \infty$, then

$$
\int_{R^{m}} c\left(x, M_{0}\right) d \mu \leq \lim _{k \rightarrow \infty} \int_{R^{m}} c\left(x, M_{k}\right) d \mu
$$


Proof. Since $H\left(M_{k}, M_{0}\right) \rightarrow 0$ and $M_{0}$ is bounded, all of the $M_{k}$ are contained in a ball $B_{r_{0}}$ of radius $r_{0}$ and centered at the origin. Let $r \geq r_{0}$ be any number. For $\delta>0$, define

$$
E(\delta)=\sup \left\{\left|c\left(x, y_{1}\right)-c\left(x, y_{2}\right)\right|: x, y_{1}, y_{2} \in B_{r},\left|y_{1}-y_{2}\right| \leq \delta\right\}
$$

Then the continuity of $c$ implies that

$$
E(\delta) \rightarrow 0 \text { as } \delta \rightarrow 0
$$

Now for $x \in B_{r}$ and each $k$, let $y_{k} \in M_{k}$ be such that

$$
c\left(x, y_{k}\right)=c\left(x, M_{k}\right)
$$

and $y_{0} \in M_{0}$ be such that $\left|y_{0}-y_{k}\right|=H\left(M_{k}, M_{0}\right)$. (Note that $y_{0}$ may also depend on $k$.) Therefore

$$
\begin{aligned}
c\left(x, M_{0}\right) & \leq c\left(x, y_{0}\right) \leq c\left(x, y_{k}\right)+\left|c\left(x, y_{k}\right)-c\left(x, y_{0}\right)\right| \\
& \leq c\left(x, y_{k}\right)+E\left(H\left(M_{k}, M_{0}\right)\right) \\
& =c\left(x, M_{k}\right)+E\left(H\left(M_{k}, M_{0}\right)\right) .
\end{aligned}
$$

Integrating over $B_{r}$, we get

$$
\int_{B_{r}} c\left(x, M_{0}\right) d \mu(x) \leq \int_{B_{r}} c\left(x, M_{k}\right) d \mu(x)+\mu\left[B_{r}\right] E\left(H\left(M_{k}, M_{0}\right)\right)
$$

Taking limit as $k \rightarrow \infty$ and using (21) and $H\left(M_{k}, M_{0}\right) \rightarrow 0$ we have

$$
\begin{aligned}
\int_{B_{r}} c\left(x, M_{0}\right) d \mu(x) & \leq \lim _{k \rightarrow \infty} \int_{B_{r}} c\left(x, M_{k}\right) d \mu(x) \\
& \leq \lim _{k \rightarrow \infty} \int_{R^{m}} c\left(x, M_{k}\right) d \mu(x) .
\end{aligned}
$$

Since $r$ is arbitrary, we conclude (20).

Proof of Theorem 3. Assumption 2(i) implies that for all $M \in \mathcal{M}$,

$$
F(M)<c_{0}
$$

For otherwise we would have $c(x, M) \equiv c_{0}$, contradicting (18). So the greatest lower bound $F_{\text {min }}$ of $F$ among all possible $M \in \mathcal{M}$ exists and satisfies

$$
F_{\min }<c_{0}
$$

Take a minimizing sequence $\left\{M_{k}\right\}_{k=1}^{\infty}$ such that $F\left(M_{k}\right) \rightarrow F_{\min }$ as $k \rightarrow \infty$. Let

$$
d\left(0, M_{k}\right)=\min \left\{\|x\|: x \in M_{k}\right\}
$$


which is the distance from the origin to $M_{k}$.

Assertion. The sequence $\left\{d\left(0, M_{k}\right)\right\}$ is bounded; that is, there is a number, say $d_{1}$, such that for all $k$,

$$
d\left(0, M_{k}\right) \leq d_{1}
$$

For otherwise, we may assume $d\left(0, M_{k}\right) \rightarrow \infty$ as $k \rightarrow \infty$. By assumption (19), for any

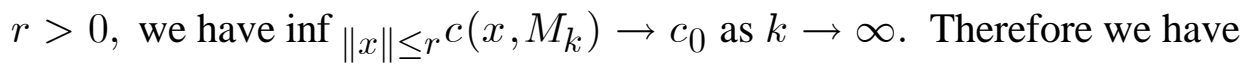

$$
\begin{aligned}
F\left(M_{k}\right) & =\int_{R^{m}} c\left(x, M_{k}\right) d \mu \\
& \geq \int_{x \in B_{r}} c\left(x, M_{k}\right) d \mu \\
& \geq \inf _{x \in B_{r}} c\left(x, M_{k}\right) \mu\left[B_{r}\right] .
\end{aligned}
$$

Taking limit as $k \rightarrow \infty$, we have $F_{\min } \geq c_{0} \mu\left[B_{r}\right]$. Since $r$ is arbitrary, we obtain $F_{\min } \geq c_{0}$, a contradiction to (23). So the assertion is proved.

Let $d_{0}$ be as in (17). By the assumptions (18) and (19) there is a number $R_{0}$ such that for all $x \in \operatorname{Spt}(\mu)$ (a compact set), we have

$$
\inf _{\|z\| \geq R_{0}-d_{0}} c(x, z) \geq \max _{\|w\| \leq d_{1}+d_{0}} c(x, w) .
$$

For each $k$, write $M_{k}=M_{k}^{\prime} \cup M_{k}^{\prime \prime}$, where

$$
\begin{aligned}
& M_{k}^{\prime}=\cup\left\{D: D \text { is a connected component of } M_{k} \text { with } d(0, D) \leq R_{0}\right\}, \\
& M_{k}^{\prime \prime}=\cup\left\{D: D \text { is a connected component of } M_{k} \text { with } d(0, D)>R_{0}\right\} .
\end{aligned}
$$

By Assumption 1(i), $M_{k}^{\prime} \in \mathcal{M}$ for each $k$. Note that if $z \in M_{k}^{\prime \prime}$, then $z \in D \subset M_{k}$ for some connected component $D$ with $d(0, D)>R_{0}$. By (17), $\operatorname{diam}(D) \leq d_{0}$. So $\|z\| \geq R_{0}-d_{0}$. At the same time, by (24) there is a $w \in M_{k}$ such that $\|w\| \leq d_{1}+d_{0}$. Therefore, by (26), for $x \in \operatorname{Spt}(\mu)$,

$$
c\left(x, M_{k}^{\prime \prime}\right) \geq c(x, w) \geq c\left(x, M_{k}\right)
$$

Consequently, we have

$$
c\left(x, M_{k}^{\prime}\right)=c\left(x, M_{k}\right), \text { for } x \in \operatorname{Spt}(\mu)
$$

which implies that $F\left(M_{k}^{\prime}\right)=F\left(M_{k}\right)$. So $\left\{M_{k}^{\prime}\right\}_{k}$ is also a minimizing sequence, with bounded 
diameters. By Blaschke's selection theorem [7, p.37], $\left\{M_{k}^{\prime}\right\}_{k}$ contains a subsequence converging to $M_{0}$ in Hausdorff distance. By Assumption 1(ii), $M_{0} \in \mathcal{M}$. By Lemma 6, $F\left(M_{0}\right) \leq \lim _{k \rightarrow \infty} F\left(M_{k}^{\prime}\right)=F_{\min }$. So $M_{0}$ minimizes $F$.

\section{Proof of Main Lemma from Section 2}

Recall $\lambda=\left(\lambda_{1}, \ldots, \lambda_{n}\right)$ and $f_{i}(\lambda)=\mu\left[D_{i}\right]$, where $D_{i}$ is defined by (8). We will have occasion to group indices together. So more generally, for $I \subset\{1,2, \ldots, n\}$, we let $\lambda_{I}=\left(\lambda_{i}\right)_{i \in I}$, and let $I^{\prime}$ be the complement of $I$ so that $I^{\prime}=\{1,2, \ldots, n\} \backslash I$. Let

$$
\begin{gathered}
D_{I}=\cup_{i \in I} D_{i}=\left\{x: c\left(x, z_{i}\right)+\lambda_{i} \leq c\left(x, z_{j}\right)+\lambda_{j}, i \in I, j \in I^{\prime}\right\} \\
f_{I}(\lambda)=\mu\left[D_{I}\right]=\sum_{i \in I} f_{i}(\lambda) .
\end{gathered}
$$

We will occasionally write $f_{I}(\lambda)$ as $f_{I}\left(\lambda_{I}, \lambda_{I^{\prime}}\right)$ and $D_{I}$ as $D_{I}\left(\lambda_{I}, \lambda_{I^{\prime}}\right)$ to emphasize the dependence on $\lambda_{I}, \lambda_{I^{\prime}}$.

Parts (i) and (ii) of the Main Lemma stated in Section 2 are direct consequences of Lemma 1 and Lemma 4 below. The proofs of these lemmas rely on several properties of $f_{i}(\lambda)$ and $f_{I}(\lambda)$ contained in other lemmas in this section.

Lemma 1. For any subset I of $\{1,2, \ldots, n\}, f_{I}$ is continuous in $\lambda_{1}, \ldots, \lambda_{n}$.

This fact can be derived from the following general fact.

Lemma 1.1. For $\gamma=\left(\gamma_{1}, \ldots, \gamma_{m}\right) \in R^{m}$ and continuous real-valued functions $g_{1}, \ldots, g_{m}$ on $R^{m}$ such that $\mu\left\{x: g_{i}(x)=\alpha\right\}=0$ for all $i$ and $\alpha$. Define $g(\lambda)=\mu\left\{x: g_{1}(x) \leq \lambda_{1}, \ldots, g_{m}(x) \leq \lambda_{m}\right\}$. Then $g$ is continuous.

Proof. We will prove the case $m=2$; the cases $m \geq 3$ are similar. Suppose $\left\{\left(a_{k}, b_{k}\right)\right\}_{k}$ is a sequence such that $\left(a_{k}, b_{k}\right) \rightarrow\left(a_{0}, b_{0}\right)$ as $k \rightarrow \infty$. We need to show that

$$
g\left(a_{0}, b_{0}\right)=\lim _{k \rightarrow \infty} g\left(a_{k}, b_{k}\right) .
$$

Define sequences $\left\{\left(a_{k}^{\prime}, b_{k}^{\prime}\right)\right\}_{k}$ and $\left\{\left(a_{k}^{\prime \prime}, b_{k}^{\prime \prime}\right)\right\}_{k}$ that squeeze $\left(a_{k}, b_{k}\right)$ as follows:

$$
a_{k}^{\prime}=\inf \left\{a_{k}, a_{k+1}, \ldots\right\}, b_{k}^{\prime}=\inf \left\{b_{k}, b_{k+1}, \ldots\right\} \text {, }
$$




$$
a_{k}^{\prime \prime}=\sup \left\{a_{k}, a_{k+1}, \ldots\right\}, b_{k}^{\prime \prime}=\sup \left\{b_{k}, b_{k+1}, \ldots\right\} .
$$

Then it is clear that componentwise $\left(a_{k}^{\prime}, b_{k}^{\prime}\right) \leq\left(a_{0}, b_{0}\right) \leq\left(a_{k}^{\prime \prime}, b_{k}^{\prime \prime}\right)$, that both components of $\left(a_{k}^{\prime}, b_{k}^{\prime}\right)$ are increasing, that both components of $\left(a_{k}^{\prime \prime}, b_{k}^{\prime \prime}\right)$ are decreasing, and both converge to $\left(a_{0}, b_{0}\right)$ as $k \rightarrow \infty$. Definethree sequences of sets

$$
\begin{aligned}
& F_{k}=\left\{x: g_{1}(x) \leq a_{k}^{\prime} \text { and } g_{2}(x) \leq b_{k}^{\prime}\right\}, \\
& G_{k}=\left\{x: g_{1}(x) \leq a_{k} \text { and } g_{2}(x) \leq b_{k}\right\}, \\
& H_{k}=\left\{x: g_{1}(x) \leq a_{k}^{\prime \prime} \text { and } g_{2}(x) \leq b_{k}^{\prime \prime}\right\}
\end{aligned}
$$

Then

$$
g\left(a_{k}^{\prime}, b_{k}^{\prime}\right)=\mu\left[F_{k}\right], g\left(a_{k}, b_{k}\right)=\mu\left[G_{k}\right], g\left(a_{k}^{\prime \prime}, b_{k}^{\prime \prime}\right)=\mu\left[H_{k}\right] .
$$

From the properties of the sequences, we see that

$$
F_{k} \subset G_{k} \subset H_{k} \text { and } g\left(a_{k}^{\prime}, b_{k}^{\prime}\right) \leq g\left(a_{k}, b_{k}\right) \leq g\left(a_{k}^{\prime \prime}, b_{k}^{\prime \prime}\right)
$$

for $k=1,2, \ldots$, and the equalities hold for $k=0$. Since $H_{k}$ is decreasing and $H_{0}=\cap H_{k}$, we have $\lim _{k \rightarrow \infty} g\left(a_{k}^{\prime}, b_{k}^{\prime}\right)=\lim _{k \rightarrow \infty} \mu\left(H_{k}\right)=\mu\left(H_{0}\right)=g\left(a_{0}, b_{0}\right)$. On the other hand, we have $F_{k}$ is increasing and

$$
\bigcup_{k} F_{k} \subset F_{0} \subset\left\{x: g_{1}(x)=a_{0}\right\} \cup\left\{x: g_{2}(x)=b_{0}\right\} \bigcup_{k} F_{k}
$$

Since the measures of both $\left\{x: g_{1}(x)=a_{0}\right\}$ and $\left\{x: g_{2}(x)=b_{0}\right\}$ are zero, it follows that $\mu\left(F_{k}\right) \rightarrow \mu\left(F_{0}\right)$, that is, $\lim _{k \rightarrow \infty} g\left(a_{k}^{\prime \prime}, b_{k}^{\prime \prime}\right)=g\left(a_{0}, b_{0}\right)$.

Consequently we have $\lim _{k \rightarrow \infty} g\left(a_{k}, b_{k}\right)=g\left(a_{0}, b_{0}\right)$. So $g$ is continuous.

\section{Proof of Lemma 1.}

Note that $f_{I}(\lambda)=\mu\left[D_{I}\right]$, where $D_{I}$ can be expressed in the form

$$
\left\{x: g_{1}(x) \leq \gamma_{1}, \ldots, g_{m}(x) \leq \gamma_{m}\right\}
$$

where $g_{i}(x)$ takes the form of $c\left(x, z_{k}\right)-c\left(x, z_{l}\right)$ and $\gamma_{i}=\lambda_{k}-\lambda_{l}$. By Lemma 1.1, $\mu\left[D_{I}\right]$ is continuous in $\gamma_{1}, \ldots, \gamma_{m}$, and so is continuous in $\lambda_{1}, \ldots, \lambda_{n}$.

Lemma 2. (a) $f_{I}\left(\lambda_{I}, \lambda_{I^{\prime}}\right)$ increases if each component of $\lambda_{I^{\prime}}$ increases or each component of $\lambda_{I}$ decreases.

(b) Assume $\mu$ is strongly positive and $0<f_{I}(\lambda)<1$. Then $f_{I}(\lambda)$ strictly increases if each component of $\lambda_{I^{\prime}}$ strictly increases or if each component of $\lambda_{I}$ strictly decreases. 
In the proof of the Lemma, we use the slightly awkward notation $\lambda_{I}^{*}$ for thepoint $\left(\lambda_{i}^{*}\right)_{i \in I}$.

Proof. For part (a), suppose $\left(\lambda_{I}, \lambda_{I^{\prime}}\right)$ is fixed and $\lambda_{I^{\prime}} \leq \lambda_{I^{\prime}}^{*}$ component-wise, then from the definition of $D_{I}$, we see $D_{I}\left(\lambda_{I}, \lambda_{I^{\prime}}\right) \subset D_{I}\left(\lambda_{I}, \lambda_{I^{\prime}}^{*}\right)$, which implies that $f_{I}\left(\lambda_{I}, \lambda_{I^{\prime}}\right) \leq f_{I}\left(\lambda_{I}, \lambda_{I^{\prime}}^{*}\right)$. Similarly, if the components of $\lambda_{I}$ each decrease, then $f_{I}(\lambda)$ decreases.

For part (b), suppose $\left(\lambda_{I}, \lambda_{I^{\prime}}\right)$ is fixed such that

$$
f_{I}\left(\lambda_{I}, \lambda_{I^{\prime}}\right)=\mu\left[D_{I}\left(\lambda_{I}, \lambda_{I}\right)\right] \in(0,1),
$$

and $\lambda_{I}<\lambda_{I}^{*}$ component-wise. From the definition of $D_{I}$ we see

$$
D_{I}\left(\lambda_{I}, \lambda_{I^{\prime}}\right) \supset D_{I}\left(\lambda_{I}^{*}, \lambda_{I^{\prime}}\right)
$$

By assumption (7) and (27), we know $D_{I}=D_{I}\left(\lambda_{I}, \lambda_{I}\right)$ must have non-empty interior, denoted by $\operatorname{Int}\left(D_{I}\right)$. Note that $(27)$ also implies that $\operatorname{Int}\left(D_{I}\right)$ cannot be the whole space $R^{m}$, therefore $\partial \operatorname{Int}\left(D_{I}\right)$ is non-empty.

Take a point $x_{0} \in \partial \operatorname{Int}\left(D_{I}\right)$. Then for any $r>0, B\left(x_{0}, r\right) \cap \operatorname{Int}\left(D_{I}\right)$ is open and nonempty, and because $\mu$ is strongly positive, we have

$$
\mu\left[B\left(x_{0}, r\right) \cap \operatorname{Int}\left(D_{I}\right)\right]>0
$$

Note that

$$
\operatorname{Int}\left(D_{I}\right)=\left\{x: \max \left\{c\left(x, z_{i}\right)+\lambda_{i}, i \in I\right\}<\min \left\{c\left(x, z_{j}\right)+\lambda_{j}, j \in I^{\prime}\right\}\right\}
$$

so

$$
\begin{aligned}
& \partial \operatorname{Int}\left(D_{I}\right) \subset \partial D_{I} \\
& =\left\{x: \max \left\{c\left(x, z_{i}\right)+\lambda_{i}, i \in I\right\}=\min \left\{c\left(x, z_{j}\right)+\lambda_{j}, j \in I^{\prime}\right\}\right\}
\end{aligned}
$$

By (31) and $\lambda_{I}<\lambda_{I}^{*}$, we may take a small $r>0$ such that if $x \in B\left(x_{0}, r\right)$, then

$$
\max \left\{c\left(x, z_{i}\right)+\lambda_{i}^{*}, i \in I\right\}>\min \left\{c\left(x, z_{j}\right)+\lambda_{j}, j \in I^{\prime}\right\} .
$$


Note that (32) implies that $x \notin D_{I}\left(\lambda_{I}^{*}, \lambda_{I^{\prime}}\right)$; so $B\left(x_{0}, r\right) \cap D_{I}\left(\lambda_{I}^{*}, \lambda_{I^{\prime}}\right)=\emptyset$. This together with (28) and (29) implies

$$
\begin{aligned}
f_{I}\left(\lambda_{I}, \lambda_{I^{\prime}}\right) & =\mu\left[D_{I}\left(\lambda_{I}, \lambda_{I^{\prime}}\right)\right] \\
& \geq \mu\left[B\left(x_{0}, r\right) \cap D_{I}\left(\lambda_{I}, \lambda_{I^{\prime}}\right)\right]+\mu\left[D_{I}\left(\lambda_{I}^{*}, \lambda_{I^{\prime}}\right)\right] \\
& \geq \mu\left[B\left(x_{0}, r\right) \cap \operatorname{Int}\left(D_{I}\right)\right]+\mu\left[D_{I}\left(\lambda_{I}^{*}, \lambda_{I^{\prime}}\right)\right] \\
& >\mu\left[D_{I}\left(\lambda_{I}^{*}, \lambda_{I^{\prime}}\right)\right] \\
& =f_{I}\left(\lambda_{I}^{*}, \lambda_{I^{\prime}}\right) .
\end{aligned}
$$

So $f_{I}$ strictly decreases if each component of $\lambda_{I}$ strictly increases. The assertion for $\lambda_{I^{\prime}}$ is proved similarly.

We write $\lambda_{I} \rightarrow \infty(-\infty)$ if each component approaches $\infty(-\infty$, respectively).

Lemma 3. If $\lambda_{I} \rightarrow \infty$, then $f_{I}(\lambda) \rightarrow 0$.

$$
\text { If } \lambda_{I} \rightarrow-\infty \text {, then } f_{I}(\lambda) \rightarrow 1 \text {. }
$$

This is obvious from the definition of $D_{I}$.

Relating to Lemma 3, we note that $D_{I}$ is well-defined even if some of the $\lambda_{i}^{\prime}$ s are replaced by $\infty$ or $-\infty$ as long as an infinity of the same sign does not appear on the both sides of any inequality. For example,

$$
\begin{aligned}
f_{1}\left(\lambda_{1}, \ldots, \lambda_{n-1}, \infty\right) & =\lim _{\lambda_{n} \rightarrow \infty} f_{1}\left(\lambda_{1}, \ldots, \lambda_{n}\right) \\
& =\mu\left[\left\{x: c\left(x, z_{1}\right)+\lambda_{1} \leq c\left(x, z_{j}\right)+\lambda_{j}, j=2, \ldots, n-1\right\}\right]
\end{aligned}
$$

Here the condition for $\lambda_{n}$ is void because $\lambda_{n}=\infty$.

Lemma 4. Suppose $\mu$ is strongly positive. For $I=\{1, \ldots k\}$ with $k=0, \ldots, n-1$, if $\lambda_{I^{\prime}}=\left(\lambda_{k+1}, \ldots \lambda_{n}\right) \in R^{n-k}$, then there exists unique $\lambda_{I}^{*}=\left(\lambda_{1}^{*}, \ldots, \lambda_{k}^{*}\right)$ determined by $\lambda_{I^{\prime}}$ such that $f_{i}\left(\lambda_{I}^{*}, \lambda_{l}\right)=\alpha_{i}$ for $i=1, \ldots, k$.

A few remarks are needed before proving Lemma 4. Suppose $I=\{1, \ldots k\}$ with $k=0, \ldots, n-1$. For $\lambda_{I^{\prime}} \in R^{n-k}$, by Lemma 4, we can take $\lambda_{I}^{*}$ depending uniquely on $\lambda_{I^{\prime}}$ such that $f_{i}\left(\lambda_{I}^{*}, \lambda_{l}\right)=\alpha_{i}$ for $i=1, \ldots, k$. Therefore, for $j=k+1, \ldots, n$, we obtain a function $F_{j}$ 
which is, roughly, the function $f_{j}$ restricted to an " $n-k$ dimensional subset"

$$
F_{j}\left(\lambda_{I^{\prime}}\right)=f_{j}\left(\lambda_{I}^{*}, \lambda_{I^{\prime}}\right), \lambda_{I^{\prime}} \in R^{n-k}
$$

The following lemma contains properties of $F_{j}$ which are needed in the proof of Lemma 4 . These properties are analogous to the results of Lemmas 1-3 for $f_{I}$. Note that if $k=n-1$, then $F_{n}\left(\lambda_{n}\right)$ is the constant $\alpha_{n}$.

Lemma 5. Suppose $\mu$ is strongly positive and $I=\{1, \ldots k\}$ with $k=0, \ldots, n-2$. For $j=k+1, \ldots, n$, the function $F_{j}$ defined by (33) satisfies

(a) $F_{j}\left(\lambda_{I^{\prime}}\right)$ is continuous in $\lambda_{I^{\prime}}$.

(b) If $\lambda_{j}$ strictly increases, or $\lambda_{i}$ strictly decreases for all $i=k+1, \ldots, n, i \neq j$, then $F_{j}\left(\lambda_{I^{\prime}}\right)$ strictly decreases. If $\lambda_{i}$ increases for some $i=k+1, \ldots, n, i \neq j$, then $F_{j}\left(\lambda_{I^{\prime}}\right)$ increases.

(c) If $\lambda_{j} \rightarrow \infty$ then $F_{j}\left(\lambda_{I^{\prime}}\right) \rightarrow 0$, and if $\lambda_{j} \rightarrow-\infty$ then $F_{j}\left(\lambda_{I^{\prime}}\right) \rightarrow 1-\sum_{i=1}^{k} \alpha_{i}$.

\section{Proof of Lemmas 4, 5.}

We prove both Lemmas together by using mathematical induction on $k$.

If $k=0$, then $I^{\prime}=\{1, \ldots, n\}$. Lemmas 4 and 5 are implied by Lemmas 1-3 above.

Assume Lemmas 4 and 5 hold for $k-1$.

Now consider the case $k$. Let $I=\{1, \ldots, k\}, I^{\prime}=\{k+1, \ldots, n\}$. Also, let $I_{-}=\{1, \ldots, k-1\}$ and $I_{+}^{\prime}=\{k+2, \ldots, n\}$.

Proof of Lemma 4. Suppose $\lambda_{I^{\prime}}=\left(\lambda_{k+1}, \ldots, \lambda_{n}\right) \in R^{n-k}$ is given. For any $\lambda_{k} \in R$, by Lemma 4 with $k-1$, there is a unique $\lambda_{I_{-}}^{*}=\left(\lambda_{1}^{*}, \ldots, \lambda_{k-1}^{*}\right)$, depending on $\left(\lambda_{k}, \lambda_{I^{\prime}}\right)$ such that $\left(\lambda_{I_{-}}^{*}, \lambda_{k}, \lambda_{I^{\prime}}\right)$ satisfies

$$
f_{i}\left(\lambda_{I_{-}}^{*}, \lambda_{k}, \lambda_{I^{\prime}}\right)=\alpha_{i}, i=1, \ldots, k-1
$$

Now look at $f_{k}\left(\lambda_{I_{-}}^{*}, \lambda_{k}, \lambda_{I^{\prime}}\right)$. By Lemma 5 (a)(b) with $k-1, f_{k}\left(\lambda_{I_{-}}^{*}, \lambda_{k}, \lambda_{I^{\prime}}\right)$ is continuous in $\left(\lambda_{k}, \lambda_{I^{\prime}}\right)$ and strictly decreasing as a function of $\lambda_{k}$. Furthermore, by Lemma 5(c) with $k-1$ and $j=k$,

$$
f_{k}\left(\lambda_{I_{-}}^{*}, \infty, \lambda_{I^{\prime}}\right)=0, \text { and } f_{k}\left(\lambda_{I_{-}}^{*},-\infty, \lambda_{I^{\prime}}\right)=1-\sum_{i=1}^{k-1} \alpha_{i}>\alpha_{k}
$$


By the intermediate value theorem, there is unique $\lambda_{k}^{*}$ such that $f_{k}\left(\lambda_{I_{-}}^{*}, \lambda_{k}^{*}, \lambda_{I^{\prime}}\right)=\alpha_{k}$. Of course, equations (34) are still satisfiedwith $\lambda_{k}$ replaced by $\lambda_{k}^{*}$. Let $\lambda_{I}^{*}=\left(\lambda_{I_{-}}^{*}, \lambda_{k}^{*}\right)$, we then have

$$
f_{i}\left(\lambda_{I}^{*}, \lambda_{I}\right)=\alpha_{i}, i=1, \ldots, k
$$

This shows Lemma 4 with $k$.

Proof of Lemma 5(a). We assume that $k \leq n-2$. From Lemma 4, since $\lambda_{I}^{*}$ is determined by $\lambda_{I^{\prime}}$, we see that the equations

$$
F_{j}\left(\lambda_{I^{\prime}}\right)=f_{j}\left(\lambda_{I}^{*}, \lambda_{I^{\prime}}\right), \quad j=k+1, \ldots, n
$$

can be considered as functions of $\lambda_{I^{\prime}}$ in $R^{n-k}$.

If $F_{j}\left(\lambda_{I^{\prime}}\right)$ were not continuous at some $\lambda_{I^{\prime}}^{0}$, then we would have a sequence $\left\{\lambda_{I^{\prime}}^{q}\right\}_{q=1}^{\infty}$ converging to $\lambda_{I^{\prime}}^{0}$ as $q \rightarrow \infty$, but $F_{j}\left(\lambda_{I^{\prime}}^{q}\right)$ does not converge to $F_{j}\left(\lambda_{I^{\prime}}^{0}\right)$. Since $\left\{F_{j}\left(\lambda_{I^{\prime}}^{q}\right)\right\}_{q}$ is bounded, some subsequence must converge. Therefore, by passing to a subsequence, we may assume that

$$
F_{j}\left(\lambda_{I^{\prime}}^{q}\right) \rightarrow l_{j} \text { but } l_{j} \neq F_{j}\left(\lambda_{I^{\prime}}^{0}\right)
$$

Claim: the sequence $\lambda_{I}^{* q}=\left(\lambda_{1}^{* q}, \ldots, \lambda_{k}^{* q}\right)$, determined by $\lambda_{I}^{q}$, must also be bounded.

For otherwise, by passing to a subsequence, we partition the index set $I$ into those indices for which $\lambda_{i}^{* q}$ approaches $\infty,-\infty$, or a finite number. That is we define sets $I_{-\infty}, I_{\infty}$ and $I_{0}$ such that as $q \rightarrow \infty$, for $i \in I_{\infty}, I_{-\infty}$, and $I_{0}$, respectively, we have

$$
\lambda_{i}^{* q} \rightarrow \infty, \lambda_{i}^{* q} \rightarrow-\infty, \text { and } \lambda_{i}^{* q} \rightarrow \lambda_{i}^{* 0} \text { (real numbers) }
$$

If $I_{\infty} \neq \emptyset$, thenby Lemma 3

$$
\sum_{i \in I_{\infty}} \alpha_{i}=f_{I_{\infty}}\left(\lambda_{I}^{* q}, \lambda_{I^{\prime}}^{q}\right) \rightarrow 0 \text { as } q \rightarrow \infty
$$


which is impossible since each $\alpha_{i}>0$. If $I_{-\infty} \neq \emptyset$, then by Lemma 3 again

$$
\sum_{i \in I_{-\infty}} \alpha_{i}=f_{I_{-\infty}}\left(\lambda_{I}^{* q}, \lambda_{I^{\prime}}^{q}\right) \rightarrow 1 \text { as } q \rightarrow \infty
$$

which contradicts the condition $\sum_{i \in I_{-\infty}} \alpha_{i}<1$.

So $\left\{\lambda_{I}^{* q}\right\}_{q}$ must be bounded. Therefore, by passing to a subsequence, we may also assume that $\lambda_{I}^{* q}$ converges (componentwise) to some $\lambda_{I}^{* 0}$.

From (37) we have as $q \rightarrow \infty$,

$$
F_{j}\left(\lambda_{I^{\prime}}^{q}\right) \rightarrow l_{j} \neq F_{j}\left(\lambda_{I^{\prime}}^{0}\right) \text { and } F_{j}\left(\lambda_{I^{\prime}}^{q}\right)=f_{j}\left(\lambda_{I}^{* q}, \lambda_{I^{\prime}}^{q}\right) \rightarrow f_{j}\left(\lambda_{I}^{* 0}, \lambda^{0}\right) .
$$

On the other hand, by taking limit in (35): $f_{i}\left(\lambda_{I}^{* q}, \lambda_{I^{\prime}}\right)=\alpha_{i}$ as $q \rightarrow \infty$, we obtain $f_{i}\left(\lambda_{I}^{* 0}, \lambda_{I^{\prime}}^{0}\right)=\alpha_{i}, i=1, \ldots, k$, which shows that $\left(\lambda_{I}^{* 0}, \lambda_{I^{\prime}}^{0}\right) \in S_{\left\{\alpha_{1}, \ldots, \alpha_{k}\right\}}$ by definition(9). By definition (36), $F_{j}\left(\lambda_{I^{\prime}}^{0}\right)=f_{j}\left(\lambda_{I}^{* 0}, \lambda_{I^{\prime}}^{0}\right)=l_{j}$, a contradiction to (38).

Proof of Lemma 5(b). To be more specific, assume $j=k+1(k \leq n-2)$. So we need to show that if $\lambda_{k+1}$ strictly increases, or $\lambda_{I_{+}^{\prime}}=\left(\lambda_{k+2}, \ldots, \lambda_{n}\right)$ strictly decrease, then $F_{k+1}\left(\lambda_{I^{\prime}}\right)$ strictly decreases. Also, if $\lambda_{i}$ increases for some $i=k+2, \ldots, n$, then $F_{k+1}\left(\lambda_{I^{\prime}}\right)$ increases.

Fix $\lambda_{I^{\prime}}=\left(\lambda_{k+1}^{1}, \lambda_{k+2}, \ldots, \lambda_{n}\right)$ and increase $\lambda_{k+1}^{1}$ to $\lambda_{k+1}^{2}>\lambda_{k+1}^{1}$. For $q=1,2$, let $\lambda_{I}^{* q}=\left(\lambda_{1}^{* q}, \ldots, \lambda_{k}^{* q}\right)$ be determined by $\left(\lambda_{k+1}^{q}, \lambda_{I_{+}^{\prime}}\right)$ as in (35).

If $\lambda_{l}^{* 1}=\lambda_{l}^{* 2}$ for some $l \in\{1, \ldots k\}$, say, $\lambda_{k}^{* 1}=\lambda_{k}^{* 2}$, then the situation is reduced to Lemma 5(b) with $k-1$, which implies that

$$
\begin{gathered}
F_{k+1}\left(\lambda_{k}^{* 1}, \lambda_{k+1}^{1}, \lambda_{I_{+}^{\prime}}\right)>F_{k+1}\left(\lambda_{k}^{* 1}, \lambda_{k+1}^{2}, \lambda_{I_{+}^{\prime}}\right) \\
F_{j}\left(\lambda_{k}^{* 1}, \lambda_{k+1}^{1}, \lambda_{I_{+}^{\prime}}\right) \leq F_{j}\left(\lambda_{k}^{* 1}, \lambda_{k+1}^{2}, \lambda_{I_{+}^{\prime}}\right), j=k+2, \ldots n,
\end{gathered}
$$

that is, $F_{k+1}$ is strictly decreases and $F_{j}$ increases for $j \geq k+2$.

So we assume that $\lambda_{l}^{* 1}$ and $\lambda_{l}^{* 2}$ are componentwise different, $l \in\{1, \ldots k\}$. In fact, we assert that

$$
\lambda_{l}^{* 1}<\lambda_{l}^{* 2} \text { for } l \in\{1, \ldots k\}
$$

If (39) does not hold, there is a $l_{0} \geq 1$ such that $\lambda_{l}^{* 1}>\lambda_{l}^{* 2}$ for $l \in I_{0}=\left\{1, \ldots, l_{0}\right\}$ and $\lambda_{l}^{* 1}$ 
$<\lambda_{l}^{* 2}$ for $l=l_{0}+1, \ldots, k$. By the Lemma $2, f_{I_{0}}\left(\lambda_{1}, \ldots, \lambda_{n}\right)$ is strictly increasing as $\lambda_{I_{0}}$ strictly decreases and as $\lambda_{I_{0}^{\prime}}=\left(\lambda_{l_{0}+1}, \ldots, \lambda_{k}, \lambda_{k+1}, \ldots, \lambda_{n}\right)$ increases, so

$$
f_{I_{0}}\left(\lambda_{I}^{* 1}, \lambda_{k+1}^{1}, \lambda_{I_{+}^{\prime}}\right)<f_{I_{0}}\left(\lambda_{I}^{* 2}, \lambda_{k+1}^{2}, \lambda_{I_{+}^{\prime}}\right)
$$

But both sides of (40) are equal to $\sum_{i \in I_{0}} \alpha_{i}$ by the definition of $\lambda_{I}^{* q}, q=1,2$; this contradiction shows that the assertion that (39) must hold is valid.

With (39) and by Lemma 2 again, we have that for all $j \geq k+2, f_{j}$ is increasing, that is,

$$
f_{j}\left(\lambda_{I}^{* 1}, \lambda_{k+1}^{1}, \lambda_{I_{+}^{\prime}}\right) \leq f_{j}\left(\lambda_{I}^{* 2}, \lambda_{k+1}^{2}, \lambda_{I_{+}^{\prime}}\right)
$$

while their sum is strictly increasing:

$$
f_{\{k+2, \ldots, n\}}\left(\lambda_{I}^{* 1}, \lambda_{k+1}^{1}, \lambda_{I_{+}^{\prime}}\right)<f_{\{k+2, \ldots, n\}}\left(\lambda_{I}^{* 2}, \lambda_{k+1}^{2}, \lambda_{I_{+}^{\prime}}\right) .
$$

Inequality (41) implies that

$$
f_{k+1}\left(\lambda_{I}^{* 1}, \lambda_{k+1}^{1}, \lambda_{I_{+}^{\prime}}\right)>f_{k+1}\left(\lambda_{I}^{* 2}, \lambda_{k+1}^{2}, \lambda_{I_{+}^{\prime}}\right)
$$

because $\sum_{i=k+1}^{n} f_{j}\left(\lambda_{I}^{* 1}, \lambda_{k+1}^{1}, \lambda_{I_{+}^{\prime}}\right)=\sum_{i=k+1}^{n} f_{j}\left(\lambda_{I}^{* 2}, \lambda_{k+1}^{2}, \lambda_{I_{+}^{\prime}}\right)=1-\sum_{i=1}^{k} \alpha_{i}$.

In summary, we see that as $\lambda_{k+1}$ strictly increases, $f_{k+1}$ strictly decreases while $f_{j}$ increases, $j \geq k+2$. The remaining case $\left(\lambda_{i}\right.$ increasing for $\left.i=k+2, \ldots n\right)$ is similar.

Proof of Lemma 5(c). Once again we assume $j=k+1(k \leq n-2)$. Recall that the definition (36)

$$
F_{k+1}\left(\lambda_{I^{\prime}}\right)=f_{k+1}\left(\lambda_{I}^{*}, \lambda_{I^{\prime}}\right)=\mu\left[D_{k+1}\right]
$$

where $D_{k+1}$ is defined using $\lambda_{1}^{*}, \ldots, \lambda_{k}^{*}, \lambda_{k+1}, \lambda_{k+2}, \ldots, \lambda_{n}$. Recall that $\lambda_{i}^{*}(i=1, \ldots, k)$ are determined by $\lambda_{j}(j=k+1, \ldots, n)$. Note that

$$
D_{k+1} \subset\left\{x: c\left(x, z_{k+1}\right)+\lambda_{k+1} \leq c\left(x, z_{k+2}\right)+\lambda_{k+2}\right\} \rightarrow \emptyset
$$

as $\lambda_{k+1} \rightarrow \infty$. Therefore, $F_{k+1}\left(\lambda_{I^{\prime}}\right) \rightarrow 0$ as $\lambda_{k+1} \rightarrow \infty$. Here we used the assumption that $k \leq n-2$, so that $k+2$ exists and $\leq n$. 
If $\lambda_{k+1} \rightarrow-\infty$, then for $i=k+2, \ldots n$, we have $F_{i}\left(\lambda_{I^{\prime}}\right)=\mu\left[D_{i}\right] \leq$ $\mu\left[\left\{x: c\left(x, z_{k+2}\right)+\lambda_{k+2} \leq c\left(x, z_{k+1}\right)+\lambda_{k+1}\right\}\right] \rightarrow 0$. Because $\sum_{i=k+1}^{n} F_{i}\left(\lambda_{I^{\prime}}\right)=1-\sum_{i \in I} \alpha_{i}$, we conclude that $F_{k+1}\left(\lambda_{I^{\prime}}\right) \rightarrow 1-\sum_{i \in I} \alpha_{i}$ as $\lambda_{k+1} \rightarrow-\infty$.

This finishes the proof of the Lemmas.

\section{Proof of Assertion from Theorem 2.}

The proof is divided into four steps.

Step 1. If $c_{0}=\infty$,then for all $i,\left\{z_{i}^{k}\right\}_{k}$ is bounded.

Step 2. If $c_{0}<\infty$,then for some $i,\left\{z_{i}^{k}\right\}_{k}$ is bounded.

Step 3. For all $i,\left\{\lambda_{i}^{k}\right\}_{k}$ can be chosen to be bounded.

Step 4. If $c_{0}<\infty$,then for all $i,\left\{z_{i}^{k}\right\}_{k}$ are bounded.

Step 1. Suppose $c_{0}=\infty$. Suppose also thatfor some $i,\left\|z_{i}^{k}\right\| \rightarrow \infty$ as $k \rightarrow \infty$. Let $r>0$ be large enough so that $\mu[B(0, r)]>1-\alpha_{i} / 2$, which implies that $\mu\left[D_{i}^{k} \cap B(0, r)\right] \geq \alpha_{i} / 2$. So

$$
\begin{aligned}
F\left(z_{1}^{k}, \ldots, z_{n}^{k}\right) & =\sum_{i=1}^{n} \int_{D_{i}^{k}} c\left(x, z_{i}^{k}\right) d \mu \\
& \geq \int_{D_{i}^{k} \cap B(0, r)} c\left(x, z_{i}^{k}\right) d \mu \\
& \geq \min \left\{c\left(x, z_{i}^{k}\right), x \in B(0, r)\right\} \alpha_{i} / 2 .
\end{aligned}
$$

In this step, assumption (i) in Theorem 2 implies $\min \left\{c\left(x, z_{i}^{k}\right), x \in B(0, r)\right\} \rightarrow \infty$ as $k \rightarrow \infty$. Therefore, $F_{\min }=\lim _{k \rightarrow \infty} F\left(z_{1}^{k}, \ldots, z_{n}^{k}\right)=\infty$. This is a contradictionto (11). So we proved that for all $i,\left\{z_{i}^{k}\right\}_{k}$ must be bounded.

Step 2.: Suppose $c_{0}<\infty$.First note that at least one of the sequences $\left\{z_{1}^{k}\right\}, \ldots,\left\{z_{n}^{k}\right\}$ will be bounded. For otherwise, we may assume that $\left\|z_{i}^{k}\right\| \rightarrow \infty$ as $k \rightarrow \infty$ for all $i$ as $k \rightarrow \infty$. Let $\epsilon>0$ be an arbitrary number, and $r>0$ be large enough so that $\mu[B(0, r)]>1-\epsilon$. Then the assumption (i) implies that for large $k, \min \left\{c\left(x, z_{i}^{k}\right), x \in B(0, r)\right\} \geq c_{0}-\epsilon$. So we have 


$$
\begin{aligned}
F\left(z_{1}^{k}, \ldots, z_{n}^{k}\right) & =\sum_{i=1}^{n} \int_{D_{i}^{k}} c\left(x, z_{i}^{k}\right) d \mu \\
& \geq \sum_{i=1}^{n} \int_{D_{i}^{k} \cap B(0, r)} c\left(x, z_{i}^{k}\right) d \mu \\
& \geq\left(c_{0}-\epsilon\right)(1-\epsilon) .
\end{aligned}
$$

It follows that $F_{\min }=\lim _{k \rightarrow \infty} F\left(z_{1}^{k}, \ldots, z_{n}^{k}\right) \geq\left(c_{0}-\epsilon\right)(1-\epsilon)$. Since $\epsilon$ is arbitrary, we get $F_{\min } \geq c_{0}$, which contradicts (11).

Step 3. As shown in Theorem 1, we may take $\lambda_{n}^{k}=0$, for all $k$. (In defining the partition, one of the $\lambda$ 's can always be specified arbitrarily.) Therefore, if $\left\{\lambda_{i}^{k}\right\}_{k}$ is not bounded for some $i$, then by passing to a subsequence, we may assume $\lambda_{i}^{k} \rightarrow \infty$ or $-\infty$. If $\lambda_{i}^{k} \rightarrow \infty$, then

$$
\alpha_{i}=\mu\left[D_{i}^{k}\right] \leq \mu\left[\left\{x: c\left(x, z_{i}^{k}\right)+\lambda_{i}^{k} \leq c\left(x, z_{n}^{k}\right)\right\}\right] \rightarrow 0, \text { as } k \rightarrow \infty
$$

which contradicts $\alpha_{i}>0$. If $\lambda_{i}^{k} \rightarrow-\infty$, then

$$
\alpha_{n}=\mu\left[D_{n}^{k}\right] \leq \mu\left[\left\{x: c\left(x, z_{n}^{k}\right) \leq c\left(x, z_{i}^{k}\right)+\lambda_{i}^{k}\right\}\right]
$$

Note that in the case $c_{0}=\infty,\left\{z_{i}^{k}\right\}_{k}$ must also be bounded then as shown in step 1; in case $c_{0}<\infty, c\left(x, z_{i}^{k}\right)<c_{0}$. So in either case, $c\left(x, z_{i}^{k}\right)$ is bounded (in $i$ and $k$ ), which implies the right hand term of (42) approaches 0 as $k \rightarrow \infty$, a contradiction. So $\left\{\lambda_{i}^{k}\right\}_{k}$ has to be a bounded sequence for each $i$.

Step 4. Suppose instead that there is an integer $n^{\prime} \geq 1$ such that $\left\|z_{i}^{k}\right\| \rightarrow \infty$ for $i \in\left\{1, \ldots, n^{\prime}\right\}$ and $z_{i}^{k} \rightarrow z_{i}^{0}$ for $i \in\left\{n^{\prime}+1, \ldots, n\right\}$ as $k \rightarrow \infty$. Define $D_{1}^{0}, \ldots, D_{n}^{0}$ as in (8) with $z_{1}^{0}=\infty, \ldots, z_{n^{\prime}}^{0}=\infty, z_{n^{\prime}+1}^{0}, \ldots, z_{n}^{0}$ with the convention $c(x, \infty)=c_{0}$ for any $x$. Then as shown in the proof of Theorem2, we have $D_{i}^{0}, i=1, \ldots, n$ satisfies condition (6). Again let $r>0$ be large enough so that $\mu[B(0, r)]>1-\epsilon$. Then

$$
\begin{aligned}
F\left(z_{1}^{k}, \ldots, z_{n}^{k}\right) & =\sum_{i=1}^{n} \int_{D_{i}^{k}} c\left(x, z_{i}^{k}\right) d \mu \\
& \geq \sum_{i=1}^{n^{\prime}} \int_{D_{i}^{k} \cap B(0, r)} c\left(x, z_{i}^{k}\right) d \mu+\sum_{i=n^{\prime}+1}^{n} \int_{D_{i}^{k}} c\left(x, z_{i}^{k}\right) d \mu
\end{aligned}
$$


Take the limit as $k \rightarrow \infty$. For the first term, use the fact that $\min \left\{c\left(x, z_{i}^{k}\right), x \in B(0, r)\right\} \rightarrow c_{0}$ for $i=1, \ldots, n^{\prime}$, and for the second term apply Fatou's lemma as in (16). We have

$$
F_{\min } \geq\left(\alpha_{1}+\ldots+\alpha_{n^{\prime}}-\epsilon\right) c_{0}+\sum_{i=n^{\prime}+1}^{n} \int_{D_{i}^{0}} c\left(x, z_{i}^{0}\right) d \mu
$$

Since $\epsilon$ is arbitrary, we get

$$
F_{\min } \geq\left(\alpha_{1}+\ldots+\alpha_{n^{\prime}}\right) c_{0}+\sum_{i=n^{\prime}+1}^{n} \int_{D_{i}^{0}} c\left(x, z_{i}^{0}\right) d \mu .
$$

We show how this contradicts the definition of $F_{\min }$. By replacing $z_{1}^{0}=\infty, \ldots, z_{n^{\prime}}^{0}=\infty$ by any $n^{\prime}$ points, say $z_{1}^{*}, \ldots, z_{n^{\prime}}^{*}$, and with the same partition $D_{i}^{0}$, we get cost

$$
\sum_{i=1}^{n^{\prime}} \int_{D_{i}^{0}} c\left(x, z_{i}^{*}\right) d \mu+\sum_{i=n^{\prime}+1}^{n} \int_{D_{i}^{0}} c\left(x, z_{i}^{0}\right) d \mu .
$$

As noted in (10), $\int_{D_{i}^{0}} c\left(x, z_{i}^{*}\right) d \mu<\alpha_{i} c_{0}$ for each $i$. So (44) is strictly smaller than the cost in (43), which contradicts $F_{\text {min. }}$. So all $\left\{z_{i}^{k}\right\}$ must be bounded.

\section{REFERENCES}

[1] Abdellaoui, T., Determination d'un couple optimal du probleme de Monge-Kantorovich, C. $R$. Acad. Sci. Paris, Serie I, 319(1994) 981-984.

[2] Braid, R.M., The optimal locations of branch facilities and main facilities with consumer search, Journal of Regional Science, 36(1996) 217-234.

[3] Brandeau, M.L. and S.S. Chiu, An overview of representative problems in location research, Mgmt. Sci. 35(1989) 645-674 .

[4] Cuesta-Albertos, J.A., Medidas de centralizacion multidimensionales (ley fuerte de los grandes numeros), Trab. Est. Invest. Operativa, 35 (1984) 3-16.

[5] Cuesta-Albertos, J.A. and C. Matran, The strong law of large numbers for $k$-means and best possible nets of Banach valued random variables, Prob. Theor. and Related Fields, 78(1988) 523-534.

[6] Cuesta-Albertos, J.A. and A. Tuero-Diaz, A characterization for the solution of the MongeKontorovitch mass transference, Stat. Prob. Letter, 16(1993) 147-152.

[7] Falconer, K.J., The Geometry of fractal sets. Cambridge University Press, Cambridge 1992. 
[8] Gangbo, Wilfrid and Robert J. McCann, The geometry of optimal transportation, Acta Mathematica, 177(1996) 113-161.

[9] Highfill, Jannett, Michael McAsey, and Libin Mou, The optimal location of two recycling centers, The Journal of Economics, 23(1997) 107-121.

[10] Ruschendorf, L., Optimal solution of multivariate coupling problems, Appl. Mathematicae, 23(1995) 325-338.

[11] Ruschendorf, L. and L. Uckelman, On optimal multivariate couplings, pp. 261-273 in book Distributions with given marginals and moment problems, ed. V. Benes and J. Stepan, Kluwer, 1997.

[12] McAsey, Michael and Libin Mou, Existence and characterization of optimal locations. Preprint, 1997.

[13] Rachev, S.T., The Monge-Kantorovich mass transference problem and its stochastic applications, Theory of Probability and Its Applications, 29 (1985) 647-676.

[14] ReVelle, Charles S. and Gilbert Laporte, The plant location problem: new models and research prospects, OR Chronicle, 44 (1996) 864-874.

[15] Tarpey, T. and B. Flury, Self-consistency: A fundamental concept in statistics, Stat. Science, 11(1996) 229-243.

[16] Thisse, Jacques-François, Location theory, regional science, and economics, J. Regional Science, 27 (1987) 519-528.

[17]Vitushkin, A.G., A proof of the upper semicontinuity of a set variation, Translation: Soviet Math. Dokl, 7(1966) 206-209.

[18] Ye, M.-H. and A.M. Yezer, Location and Spatial Pricing for Public Facilities, Journal of Regional Science, 32 (1992) 143-154. 